\title{
How COVID-19 has changed the unselected medical take: an observational study
}

\author{
Authors: Kai Man Alexander Ho, ${ }^{\mathrm{A}}$ Ananthi Anandhakrishnan, ${ }^{\mathrm{B}}$ Arun Mahay, ${ }^{\mathrm{C}}$ Yiwen Soo, ${ }^{\mathrm{C}}$ Laurence B Lovat ${ }^{\mathrm{D}}$ and \\ Andrew P RochfordE
}

\section{Introduction \\ COVID-19 has had a profound effect on the NHS. Little information has been published as to how the unselected medical take has been affected.}

\section{Methods}

We retrospectively reviewed patients who were referred to general medicine during March 2020. We compared clinical outcomes of patients with and without COVID-19.

Results

814 patients were included, comprising 777 unique patients. On average, 26 patients were admitted per day. $38 \%$ of admitted patients were suspected of COVID-19, with greater numbers of COVID-19 patients in the second half compared to the first half of the month $(p<0.001)$. Logistic regression analyses showed suspected COVID-19 was an independent predictor for inpatient mortality (odds ratio $[O R]=6.09$, $\mathrm{p}<0.001)$ and 30 -day mortality $(O R=4.66, p<0.001)$.

\section{Conclusions}

COVID-19 patients had worse clinical outcomes and increased healthcare use compared to non-COVID-19 patients. Our study highlights some of the challenges in healthcare provision faced during this pandemic.

KEYWORDS: COVID-19, medical take, medical admissions, SARS-CoV-2

DOI: $10.7861 /$ clinmed.2020-0483

\section{Introduction}

The COVID-19 pandemic, which has led to unprecedented numbers of patients needing critical care, has faced the NHS with 'a challenge unlike any other in its 70 years of existence' 1,2 To

Authors: ${ }^{A}$ gastroenterology registrar and clinical research fellow, Newham University Hospital, London, UK and University College London, London, UK; Binternal medicine trainee, Newham University Hospital, London, UK; ' Cfoundation year-2 doctor, Newham University Hospital, London, UK; Dprofessor of gastroenterology and biophotonics, University College London, London, UK; E' Consultant gastroenterologist, Newham University Hospital, London, UK mitigate this, NHS England advised trusts to increase critical care and inpatient capacity, as well as staffing and respiratory support capacity. ${ }^{3}$ Likewise Newham University Hospital, part of Barts Health NHS Trust, made similar provisions by reconfiguring wards and redeploying staff across the Trust. ${ }^{4}$

To our knowledge, the impact of the COVID-19 pandemic on the unselected medical take has not been investigated. Our aim therefore is to chart how the COVID-19 pandemic has affected medical admissions to and patient outcomes in a district general hospital as the pandemic progressed during the month of March a month in which cumulative UK cases rose from 69 to 38,476 and UK deaths from 0 to $4,428 .{ }^{5}$

\section{Patients and methods}

This was designed as a retrospective observational study. We screened all patients who were admitted via the unselected medical take from 1 March to 31 March 2020 at Newham University Hospital, an NHS district general hospital located in a socially deprived area of East London, using admissions lists. Patient health records were interrogated using the hospital's electronic health record system (Cerner EHR, North Kansas City MO, USA). We collected data on patient demographics, comorbidities, smoking history, admitting diagnosis, length of stay, mortality and requirement for ventilatory support. Obesity was defined as a body mass index (BMI) $\geq 30$, and chronic kidney disease (CKD) was defined as an estimated glomerular filtration rate (eGFR) of $<60 \mathrm{ml} / \mathrm{min} / 1.73 \mathrm{~m}^{2}$ on at least two occasions, in the absence of an acute insult. We subsequently grouped admitting diagnosis by specialty, eg myocardial infarction as cardiology. To be classed as 'geriatrics', a presenting complaint concerned a patient aged over 70 years with multidisciplinary issues such as falls, delirium or reduced mobility.

Patients who remained in hospital at the time of manuscript preparation were excluded from length of stay calculations and separately documented. In addition, we recorded all available SARS-CoV-2 reverse transcriptase polymerase chain reaction (RT-PCR) swab test results for patients included in the study. Samples taken within 48 hours of arrival to hospital were deemed as admission samples. Samples taken after this timeframe were deemed as inpatient samples.

In line with NHS Health Research Authority guidance ${ }^{6}$ no patient consent or research ethics committee approval was sought for this observational study. 


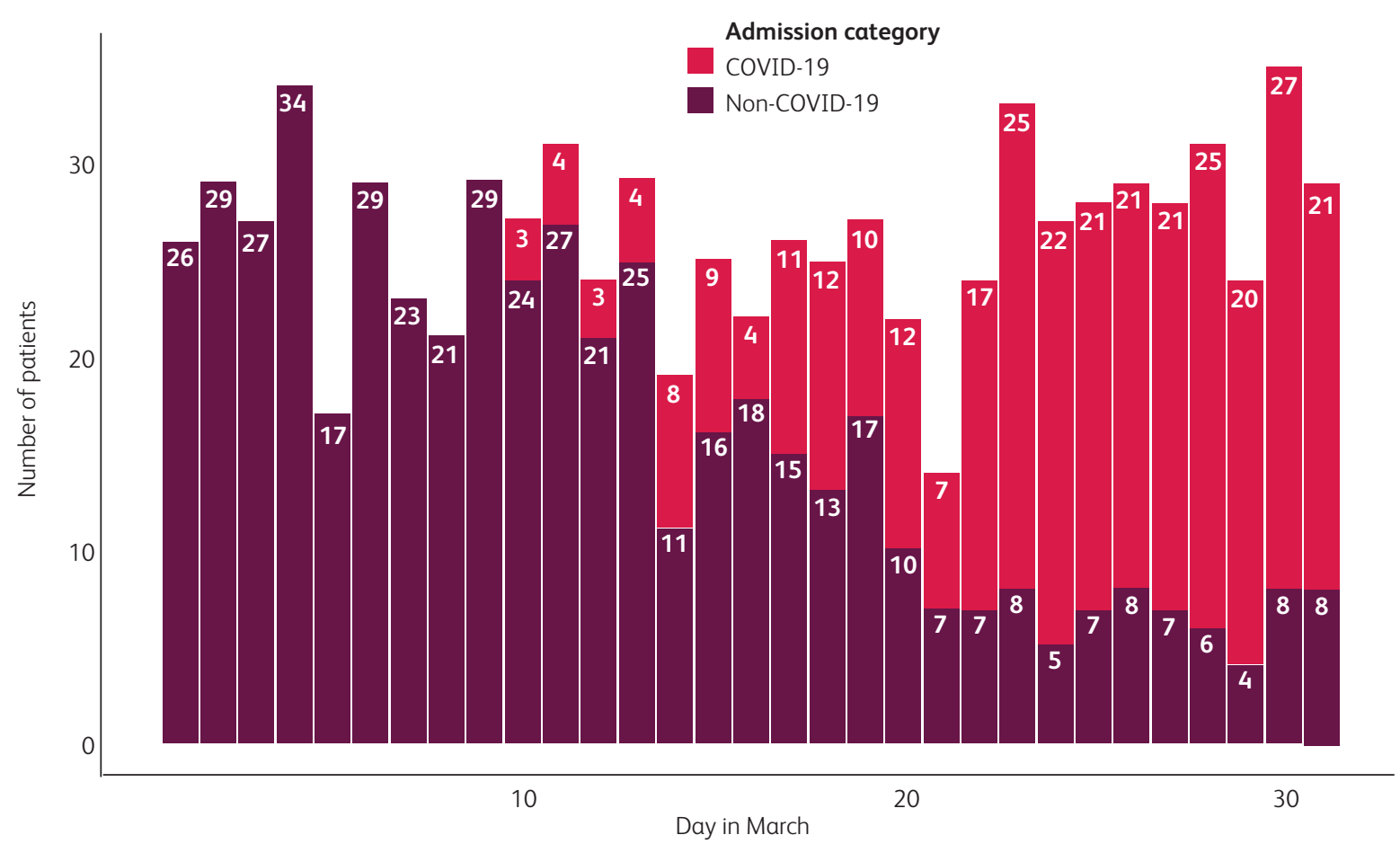

Fig 1. Number of admissions by day in March, categorised by whether the admission was COVID-19-related or not.

\section{Statistical analyses}

Data were collected using a Microsoft Excel spreadsheet over the month of May 2020 (Redmond WA, USA). Data analysis and statistical plots were produced using R: a Language and Environment for Statistical Computing software, version 4.0.2 (Vienna, Austria). Descriptive statistics with frequency and percentages were used for demographic data, admitting diagnosis and inpatient mortality. Median and interquartile range was used for age and length of stay. Differences between cohorts were analysed using the Mann-Witney $U$ test for continuous, non-parametric variables and Chi-Squared test or Fisher's Exact Test for categorical variables. We also performed univariate logistic regression modelling to identify individual factors associated with inpatient and 30-day mortality. Individual factors with a $\mathrm{p}$-value $\leq 0.10$ in univariate regression were entered into a multivariate logistic regression model to identify independent predictors of inpatient and 30-day mortality. Effect measures are presented as odds ratios (OR) and $95 \%$ confidence intervals (CI), with a $p$-value of $\leq 0.05$ taken as significant, unless otherwise stated. Patients were analysed as intention to treat, hence if a nonCOVID-19 patient subsequently tested positive for COVID-19, they remained in the non-COVID-19 cohort for analysis.

\section{Results}

In total there were 814 unselected medical admissions in March 2020. 37 patients were admitted twice in this period, meaning 777 individual patients were admitted overall. $58 \%$ of the patients were male.

\section{Number of patients admitted}

Fig 1 shows the number of admissions by day in March, as well as whether they were suspected to be COVID-19-related or not. The mean number of patients admitted per day was 26 , with a standard deviation (SD) of 4.6. Similar numbers were admitted in the first half (1-15 March) of the month (mean 26; SD 4.42) compared to the second half of the month (mean 26.5; SD 4.93). The lowest number of patients admitted on a single day was 21 March, where 14 patients were admitted. Except for this day, the admission numbers on all other days were within 2 SD of the mean. Although 21 March was a Saturday, no clear weekend effect of reduced admission numbers could be seen in our data.

The first suspected COVID-19 related admission occurred on 10 March, when three patients were admitted. The highest number recorded was 27 on the 30 March. In contrast, 4 March saw the highest number of non-COVID-19 patients admitted (34), although this decreased to single figures in the last week of March, with only four non-COVID-19 patients admitted on 29 March. Moreover, there was a gradual increase in the proportion of patients with COVID-19 as March wore on, with a decrease in non-COVID-19 patients. There was a significant difference in the proportion of COVID-19 and non-COVID-19 patients between the 1-15 March and 16-31 March $\left(X^{2}=279.98, d f=1, p<0.001\right)$.

\section{Admitting diagnosis}

Fig 2 shows the specialty relating to the admitting diagnosis during the month of March. 307 (38\%) patients were admitted with suspected COVID-19. The other major admitting diagnoses fell under cardiology (102; 13\%), non-COVID-19 respiratory (95; $12 \%)$, gastroenterology $(82 ; 10 \%)$ and geriatrics $(78,9.6 \%)$.

\section{Comparison between suspected COVID-19 and non-COVID-19 outcomes}

Table 1 summarises the demographics and clinical outcomes of suspected COVID-19 and non-COVID-19 patients. Suspected COVID-19 patients were more likely to be older $(p=0.004)$, be 


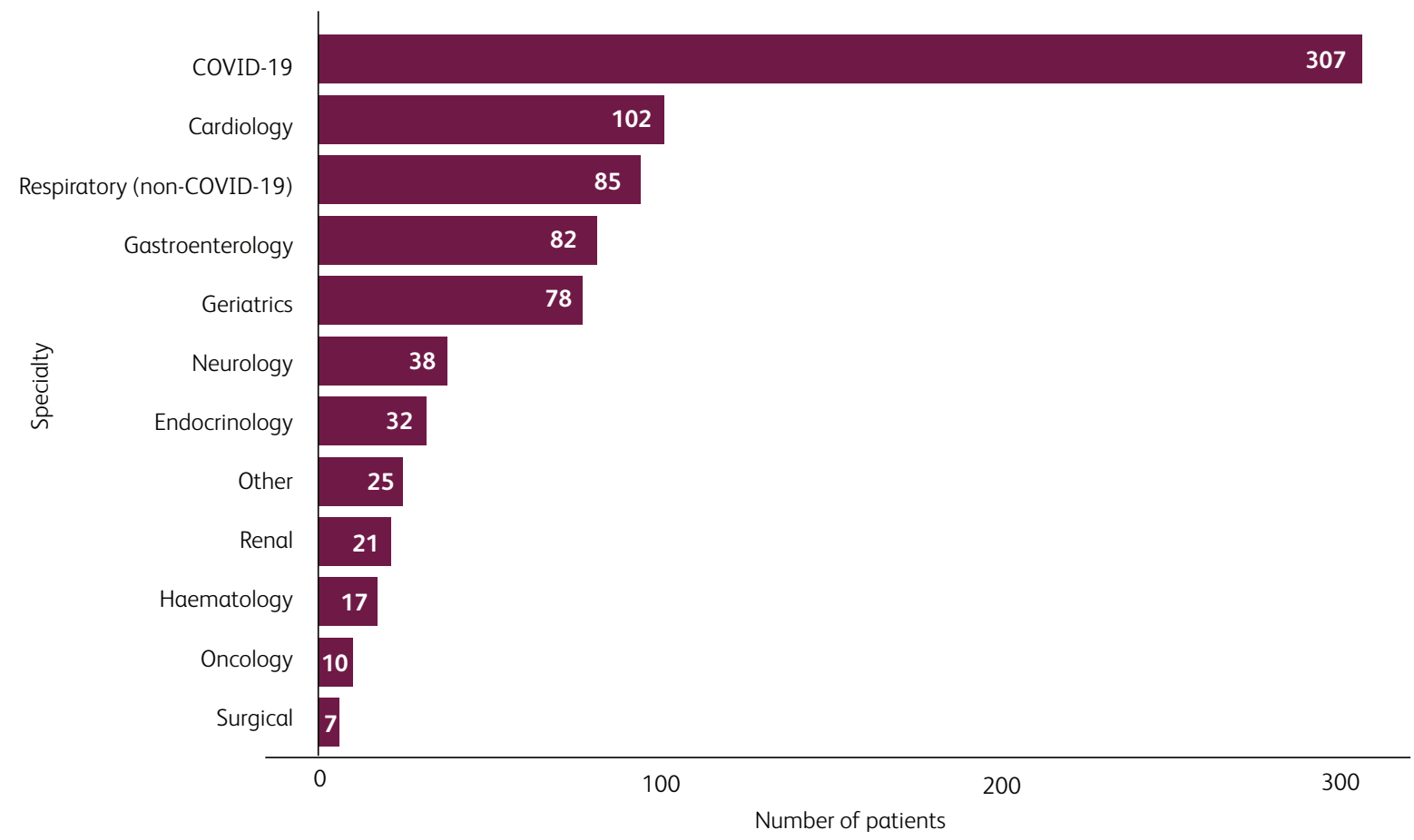

Fig 2. Admitting diagnoses during the month of March grouped by specialty.

male $(p<0.001)$ and of Black or Other ethnicity rather than White (overall $p<0.001$ ). 194/307 (63\%) patients who were admitted with suspected COVID-19 ended up with a positive admission SARS-CoV-2 swab test. In addition, we identified 18 patients who were admitted for non-COVID-19 reasons but subsequently tested positive for SARSCoV-2 in hospital. Suspected COVID-19 patients were more likely to be diabetic $(p=0.001)$ and/or hypertensive $(p=0.003)$ but less likely to have a smoking history $(p=0.002)$. Broadly speaking, patients admitted with COVID-19 had worse clinical outcomes compared to patients admitted with a non-COVID-19 diagnosis. This included a higher median length of stay $(p<0.001)$, a higher inpatient and 30-day mortality (both $p<0.001$ ) as well as a greater requirement for non-invasive ventilation and intubation (both $\mathrm{p}<0.001$ ).

Univariate logistic regression analysis was initially used to select patient factors associated with inpatient and 30-day mortality (see supplementary material S1). Subsequently, multivariate logistic regression analysis was carried out to assess the effect of age, gender, suspected COVID-19 status, diabetes mellitus, hypertension, chronic kidney disease and smoking history on mortality (see supplementary material S2). Increasing age and suspected COVID-19 status were both independent predictors for inpatient mortality, although the effect was stronger for suspected COVID-19 (suspected COVID-19: OR 6.09, 95\% CI 3.78-10.1; age: OR 1.07, 95\% CI 1.05-1.08). A similar pattern was also noted for 30-day mortality (suspected COVID-19: OR 4.66, 95\% CI 2.99-7.38; age: OR 1.06, 95\% CI 1.05-1.08).

Comparison between positive and negative SARSCoV-2 patients swab test results in suspected COVID-19 patients

We were also interested in investigating whether there were differing clinical outcomes between suspected COVID-19 patients who subsequently had a positive and a negative SARS-CoV-2 swab test (see supplementary material S3). Notably, 25 patients (22\%) who tested negative on admission subsequently had a positive SARSCoV-2 swab test as an inpatient, although only 50/113 patients $(44 \%)$ underwent further testing. Our data show that there was a significant difference in median length of stay ( 6 days compared to 4 days, $p=0.006$ ) and those remaining as inpatient ( 4 patients compared to 1 patient, $p=0.031)$. Patients testing positive also were more likely to be intubated ( $16 \%$ compared to $9 \%, p=0.04$ ) and be hypertensive ( $64 \%$ compared to $50 \%, p=0.019)$.

\section{Discussion}

We believe our study is the first to specifically investigate the effect of the COVID-19 pandemic on the unselected medical take in an NHS hospital. Perhaps surprisingly, our data demonstrate that the number of admissions during the month of March remained constant. However, the proportion of patients admitted with COVID-19 increased dramatically towards the end of March. This was compensated by a decrease in patients being admitted onto the medical take with other conditions. There have been several reports of altered health behaviour during the month of March, such as reduction in emergency department attendances, reduction in emergency hospital admissions and doubling of calls to NHS $111 .{ }^{7}$ A study demonstrated a $40 \%$ fall in patients admitted for acute coronary syndrome from mid-February to late-March 2020, with a greater decline for non-ST elevation myocardial infarctions (NSTEMI) compared to STEMI. ${ }^{8}$ In contrast, a report suggested no change to ambulance demand for both myocardial infarctions or cerebrovascular disease. ${ }^{9}$ It is likely that even though there may have been a drop in absolute numbers attending the hospital, a higher proportion of patients requiring hospitalisation counterbalanced the trend. 
Table 1. Clinical outcomes between suspected COVID-19-related and non-COVID-19-related admissions

\begin{tabular}{|c|c|c|c|}
\hline & $\begin{array}{l}\text { Suspected COVID-19 } \\
n=307\end{array}$ & $\begin{array}{l}\text { Non-COVID-19 } \\
\mathrm{n}=507\end{array}$ & p-value \\
\hline Age, years, median (IQR) & $63(26)$ & $58(34)$ & 0.004 (MWUT) \\
\hline Male, n (\%) & $202(66)$ & $264(52)$ & $<0.001$ \\
\hline $\begin{array}{l}\text { Ethnicity, } \mathrm{n} \text { (\%) } \\
\text { White (British/Irish/Other) } \\
\text { Black (African/Caribbean/Other) } \\
\text { Asian (Bangladeshi/Indian/Pakistani/Other) } \\
\text { Other (including unknown) }\end{array}$ & $\begin{array}{l}64(21) \\
79(26) \\
112(36) \\
52(17)\end{array}$ & $\begin{array}{l}185(36) \\
83(16) \\
185(36) \\
54(11)\end{array}$ & $<0.001$ \\
\hline Diabetes mellitus, n (\%) & $142(46)$ & $175(35)$ & 0.001 \\
\hline Hypertension, n (\%) & $180(59)$ & $242(48)$ & 0.003 \\
\hline Chronic kidney disease, n (\%) & $80(26)$ & $103(20)$ & 0.069 \\
\hline Obesity, n (\%) & $95(31)$ & $125(25)$ & 0.061 \\
\hline Chronic respiratory disease, $\mathrm{n}(\%$ ) & $95(31)$ & $140(28)$ & 0.349 \\
\hline Smoking history, n (\%) & $89(29)$ & $202(40)$ & 0.002 \\
\hline LOS, days, median (IQR) & $5(8)$ & $3(6)$ & $<0.001$ (MWUT) \\
\hline Still inpatient, n (\%) & $5(2)$ & $1(0)$ & 0.031 (FET) \\
\hline Inpatient mortality, n ( \%) & $86(28)$ & $32(6)$ & $<0.001$ \\
\hline 30-day mortality, n (\%) & $90(29)$ & $43(8)$ & $<0.001$ \\
\hline Non-invasive ventilation, n (\%) & $80(26)$ & $1(0)$ & $<0.001$ \\
\hline Intubation, $\mathrm{n}(\%$ ) & $42(14)$ & $1(0)$ & $<0.001$ \\
\hline
\end{tabular}

$\mathrm{FET}=$ Fisher's exact test; IQR = interquartile range; $\mathrm{LOS}=$ length of stay; MWUT = Mann-Whitney $U$ test.

Our data suggest firstly that comorbidities such as diabetes mellitus and hypertension were more common in COVID-19 compared to non-COVID-19 patients, although we found that a smoking history was more common in non-COVID-19 patients. Furthermore, there were worse clinical outcomes for patients admitted with COVID-19 compared to non-COVID-19 patients. Our multivariate logistic regression modelling suggested statistically significant associations in predicting inpatient and 30-day mortality, even when the effects of age, gender and comorbidities were factored in. Our findings are broadly in line with several larger studies which have been published looking at outcomes of COVID-19 patients in hospital. A UK multicentre series of 20,133 patients found that the median age of admission was 73 and $60 \%$ of patients were male..$^{10}$ Increasing age, male gender, diabetes, hypertension, cardiovascular disease and chronic respiratory disease were all associated with worse outcomes. The mortality rate was $26 \%$, although $34 \%$ were still inpatient at time of reporting. $16 \%$ were treated with non-invasive ventilation, and $10 \%$ were intubated. Meanwhile an American study had an intubation rate of $12 \%$ and mortality rate of $21 \%$, while a Chinese study had $14 \%$ of patients treated with non-invasive ventilation, an intubation rate of $17 \%$ and a mortality rate of $28 \% .{ }^{11,12}$ One stark contrast is that compared with the UK series, despite a younger cohort in our population, there was a similar mortality rate. Notably, it has been reported that the London Borough of Newham recorded the highest COVID-19 mortality rate in England and Wales. ${ }^{13} 78 \%$ of residents are from ethnic minorities, and there are likely to be further socioeconomic factors which led to worse outcomes in our patients. ${ }^{14,15}$ Although we were unable to demonstrate a clear relationship between mortality and ethnicity, a recent study of 1,996 COVID-19 patients based in our Trust showed that Black and Asian patients had higher mortality even after adjustment for age and sex, as well as admission to intensive care and requirement for intubation. ${ }^{16}$ Moreover, while in our study intubation rates were similar with published studies, we had a higher rate of non-invasive ventilation use. ${ }^{10-12}$ This is probably because at the beginning of the pandemic there was limited intensive care capacity, and non-invasive ventilation was often trialled first. These statistics further demonstrate how the COVID-19 pandemic created an unprecedented burden on healthcare resources and offers a glimpse as to how quickly and drastically the NHS had to adapt in response. As an example, workforce changes in our hospital included lengthening to 12.5 hour shifts for consultants and junior doctors, equal staffing numbers during the working week and weekend and redeployment of doctors from other Trust sites and research to our hospital.

Although our data found that patients with suspected COVID-19 testing positive for SARS-CoV-2 had a higher median length of stay and likelihood for intubation compared with patients testing negative, there was no difference in other outcomes such as mortality. In our cohort $22 \%$ of patients who had suspected COVID-19 but a negative admission SARS-CoV-2 test subsequently tested positive as an inpatient. However, only $44 \%$ of patients underwent repeat testing, and an early study from Wuhan found that $63 \%$ of hospitalised patients had negative initial tests. ${ }^{17}$ While it is entirely possible that nosocomial spread may be a factor, it is more likely that false negative tests were the culprit. A preprint 
systematic review consisting of five studies showed false negative rates of $2 \%$ to $29 \%$ for the SARS-CoV-2 RT-PCR test, although there was considerable heterogeneity in sensitivity estimates. ${ }^{18,19}$ A sensitivity of $70 \%$ for a SARS-CoV-2 RT-PCR test is felt to be a reasonable estimate, given at present there is no clear reference standard which RT-PCR tests are measured against. ${ }^{18}$ This therefore demonstrates the need to not be over-reliant on initial swab test results, take a repeat sample and to treat clinically if COVID-19 is suspected.

\section{Study limitations}

This was a single centre retrospective observational study and hence may not be representative of the wider UK population. Secondly, we did not investigate all confounders which may influence clinical outcomes, such as cardiovascular disease. Our study also excluded patients who were seen by the emergency department and then discharged, hence only more serious cases of COVID-19 requiring hospitalisation were included in our dataset. There is a delay in recording out of hospital mortality on the hospital systems; our quoted mortality figures may therefore underestimate the true mortality figure.

\section{Conclusions}

Our study highlights how the unselected medical take in our hospital has changed during March 2020 as the COVID-19 pandemic progressed. Patients admitted with COVID-19 typically suffered worse clinical outcomes compared with patients admitted for non-COVID-19 reasons, with greater healthcare demands. It serves as a reminder as to how severe COVID-19 can be for the individual patient, and the need for vigilance if and when COVID-19 cases rise again due to a second peak.

\section{Supplementary material}

Additional supplementary material may be found in the online version of this article at www.rcpjournals.org/clinmedicine: S1 - Univariate logistic regression analysis of baseline and clinical characteristics associated with inpatient and 30-day mortality. S2 - Multivariate logistic regression of baseline and clinical characteristics associated with inpatient and 30-day mortality. S3 - Subgroup analyses of all suspected COVID-19 patients and their admission SARS-CoV-2 RT-PCR swab test results.

\section{Acknowledgements}

The authors would like to thank Xosé Couto Parada, chief healthcare scientist in virology at Bart's Health NHS Trust for assistance with the technical aspects of the SARS-CoV-2 PCR assay.

\section{References}

1 Berlin DA, Gulick RM, Martinez FJ. Severe Covid-19. N Engl ] Med 2020, in press (doi: 10.1056/NEJMcp2009575).
2 Hunter DJ. Covid-19 and the stiff upper lip - the pandemic response in the United Kingdom. N Engl J Med 2020;382:e31.

3 Stevens S, Pritchard A. Next steps on NHS response to COVID-19. Letter from Sir Simon Stevens and Amanda Pritchard. Available from www.england.nhs.uk/coronavirus/publication/next-steps-onnhs-response-to-covid-19-letter-from-simon-stevens-and-amandapritchard [Accessed 28 May 2020].

4 Willan J, King AJ, Jeffery K et al. Challenges for NHS hospitals during Covid-19 epidemic. BMJ 2020;368:m1117.

5 Public Health England. Coronavirus (COVID-19) in the UK. https://coronavirus.data.gov.uk/ [Accessed 14 September 2020].

6 NHS Health Research Authority. Is my study research? www.hradecisiontools.org.uk/research/redirect.html [Accessed 5 June 2020].

7 Roxby P. Coronavirus: A\&E visits drop sharply as calls to 111 double. BBC News, 9 April 2020. www.bbc.co.uk/news/health52232941 [Accessed 29 May 2020].

8 Mafham MM, Spata E, Goldacre R et al. COVID-19 pandemic and admission rates for and management of acute coronary syndromes in England. Lancet 2020;396:381-89.

9 Holmes JL, Brake S, Docherty $\mathrm{M}$ et al. Emergency ambulance services for heart attack and stroke during UK's COVID-19 lockdown. Lancet 2020;395:e93-4.

10 Docherty AB, Harrison EM, Green CA et al. Features of 20133 UK patients in hospital with Covid-19 using the ISARIC WHO Clinical Characterisation Protocol: prospective observational cohort study. BMJ 2020;369:m1985.

11 Richardson S, Hirsch JS, Narasimhan M et al. Presenting characteristics, comorbidities, and outcomes among 5700 patients hospitalized With COVID-19 in the New York City Area. JAMA 2020;323:2052.

12 Zhou F, Yu T, Du R et al. Clinical course and risk factors for mortality of adult inpatients with COVID-19 in Wuhan, China: a retrospective cohort study. Lancet 2020;395:1054-62.

13 Mohdin A. 'Every day I hear about a Covid-19 death': life in the UK's worst-affected area. The Guardian, 1 May 2020. www.theguardian.com/uk-news/2020/may/01/covid-19-coronavirusnewham-london-uk-worst-affected-area [Accessed 27 May 2020].

14 Pareek M, Bangash MN, Pareek N et al. Ethnicity and COVID-19: an urgent public health research priority. Lancet 2020;395:1421-2.

15 Khunti K, Singh AK, Pareek M et al. Is ethnicity linked to incidence or outcomes of Covid-19? BMJ 2020;369:m1548.

16 Apea VJ, Wan YI, Dhairyawan R et al. Ethnicity and outcomes in patients hospitalised with COVID-19 infection in East London: an observational cohort study. medRxiv 2020; 2020.06.10.20127621.

$17 \mathrm{Li} \mathrm{Y,} \mathrm{Yao} \mathrm{L,} \mathrm{Li} \mathrm{]} \mathrm{et} \mathrm{al.} \mathrm{Stability} \mathrm{issues} \mathrm{of} \mathrm{RT-PCR} \mathrm{testing} \mathrm{of} \mathrm{SARS-}$ CoV-2 for hospitalized patients clinically diagnosed with COVID-19. J Med Virol 2020;92:903-8.

18 Woloshin S, Patel N, Kesselheim AS. False negative tests for SARS CoV-2 infection - challenges and implications. N Engl J Med 2020;383:e38.

19 Arevalo-Rodriguez I, Buitrago-Garcia D, Simancas-Racines D et al. False-negative results of initial RT-PCR assays for COVID-19: a systematic review. medRxiv 2020; 2020.04.16.20066787.

Address for correspondence: Dr Kai Man Alexander Ho, Division of Surgery and Interventional Science, University College London, Charles Bell House, 43-45 Foley Street, London W1W 7TY, UK.

Email: alexander.ho@ucl.ac.uk 